\title{
Rho Guanine Nucleotide Exchange Factor
}

\section{2}

National Cancer Institute

\section{Source}

National Cancer Institute. Rho Guanine Nucleotide Exchange Factor 12. NCI Thesaurus. Code $C 97438$.

Rho guanine nucleotide exchange factor 12 (1544 aa, 173 kDa) is encoded by the human ARHGEF12 gene. This protein plays a role in the regulation of Rho-family GT Paseand G-protein coupled receptor-mediated signal transduction. 